\title{
Period change investigation of the low mass ratio contact binary BO Ari
}

\author{
W. Kriwattanawong ${ }^{\mathrm{a}, \mathrm{b}, *}$, O. Tasuya ${ }^{\mathrm{a}}$, P. Poojon ${ }^{\mathrm{a}}$ \\ ${ }^{a}$ Department of Physics and Materials Science, Faculty of Science, Chiang Mai \\ University, Chiang Mai, 50200, Thailand \\ ${ }^{b}$ National Astronomical Research Institute of Thailand, Chiang Mai, 50200, Thailand
}

\begin{abstract}
A photometric study and period change analysis for the A-type low mass ratio contact binary $\mathrm{BO}$ Ari is presented. The $B V R$ light curves were fitted by using the Wilson-Devinney method. The photometric solution yields a low mass ratio of $q=0.1754( \pm 0.0016)$ with a contact degree of $f=27.72 \%( \pm 2.37 \%)$. We found a long-term orbital period decrease at a rate of $d P / d t=-3.49 \times 10^{-7} \mathrm{~d} \mathrm{yr}^{-1}$. This result indicates that the system is undergoing mass transfer from the primary component to the secondary with a mass transfer rate of $\dot{m}_{1} / m_{1}=-7.77 \times 10^{-8} \mathrm{yr}^{-1}$. With the period decrease, the inner and outer critical Roche surfaces will tighten and cause the degree of contact to increase. Therefore, BO Ari may evolve into a deeper contact system.
\end{abstract}

Keywords: stars: binaries: close, stars: binaries: eclipsing, stars: individual (BO Ari)

\section{Introduction}

A system consisting of two stars close enough to embedded in a common envelope, is called contact binary. These systems are classified as A-type for a system in which the more massive component is clipsed by the other one at primary minimum, or W-type for the opposite case (Binnendijk, 1970). Typically, the A-type systems tend to be hotter (Yakut and Eggleton, 2005)

*Corresponding author Tel.:+66 5394 3367; Fax.: +66 53943445

Email address: wichean.k@cmu.ac.th (W. Kriwattanawong)

Preprint submitted to New Astronomy

September 3, 2015

(C) 2015. This manuscript version is made available under the Elsevier user license http://www.elsevier.com/open-access/userlicense/1.0/ 
and have longer orbital periods (e.g. Rucinski and Duerbeck, 1997; Gazeas and Niarchos, 2006; Terrell, Gross and Cooney, 2012) than the W-type systems. The more massive component of the contact system is generally a main sequence star, while the less massive one is oversized due to its advanced evolutionary stage (Stępień, 2006).

Many researches found orbital period variations, explained by mass exchange between the components (Singh and Chaubey, 1986; Pribulla, 1998). A long-term period decrease is usually interpreted as the system is undergoing a process of mass transfer from more massive component to the less massive one and/or angular momentum loss (AML) by magnetic breaking (e.g. Vilhu, 1981; Rucinski, 1982). The result of an orbital period decrease is a deeper contact degree (e.g. Christopoulou et al., 2011; Yang, 2011). On the other hand, systems with a period increase, are predicted by the thermal relaxation oscillation (TRO) theory (e.g. Lucy, 1976; Robertson and Eggleton, 1977), to evolve into TRO cycle (e.g. Li and Qian, 2013; Liu et al., 2014). The shorter-term cyclic period change can be explained by the light time effect (LTE) due to a third body orbit around the eclipsing pair in the triple system (Irwin, 1952; Pribulla and Rucinski, 2006).

BO Ari $\left(\operatorname{RA}(2000)=02^{\mathrm{h}} 12^{\mathrm{m}} 08^{\mathrm{s}} .78\right.$, Dec. $\left.(2000)=27^{\circ} 08^{\prime} 18^{\prime \prime} .2\right)$, was identified as EW type binary by Nicholson and Varley (2006) with the first measured light minimum of HJD $=2451479.6566$ and the orbital period of $0.3182 \mathrm{~d}$. The revised period was obtained to be $0.3181963 \mathrm{~d}$ by Acerbi, Barani and Martignoni (2011). BO Ari was classified as an A-type system. Acerbi, Barani and Martignoni (2011) reported a mass ratio of $q=0.1889$ and fillout of $f=58.7 \%$. Furthermore, $B_{\text {Tycho }}=10.664$ and $V_{\text {Tycho }}=10.083$ for BO Ari were reported in the Tycho 2 catalogue (Høg et al., 2000).

This study presents $B V R$ observations for $\mathrm{BO}$ Ari and investigation on the orbital period change, cyclic vairation and an analysis of the photometric light curve. Section 2 presents the photometric observations. An analysis for long-term orbital period changes and cyclic vairations is described in Section 3. Section 4 presents a model fit to the light curve and derivation of the system parameters. Finally, Section 5. contains discussion and conclusions.

\section{Observations}

BO Ari was observed at Sirindhorn Observatory, Chiang Mai University on November 21, 24 and 26, 2011. The 0.5-m telescope, equipped with a SBIG ST10-XME CCD, was used for $B V R$ filter bands with an exposure 

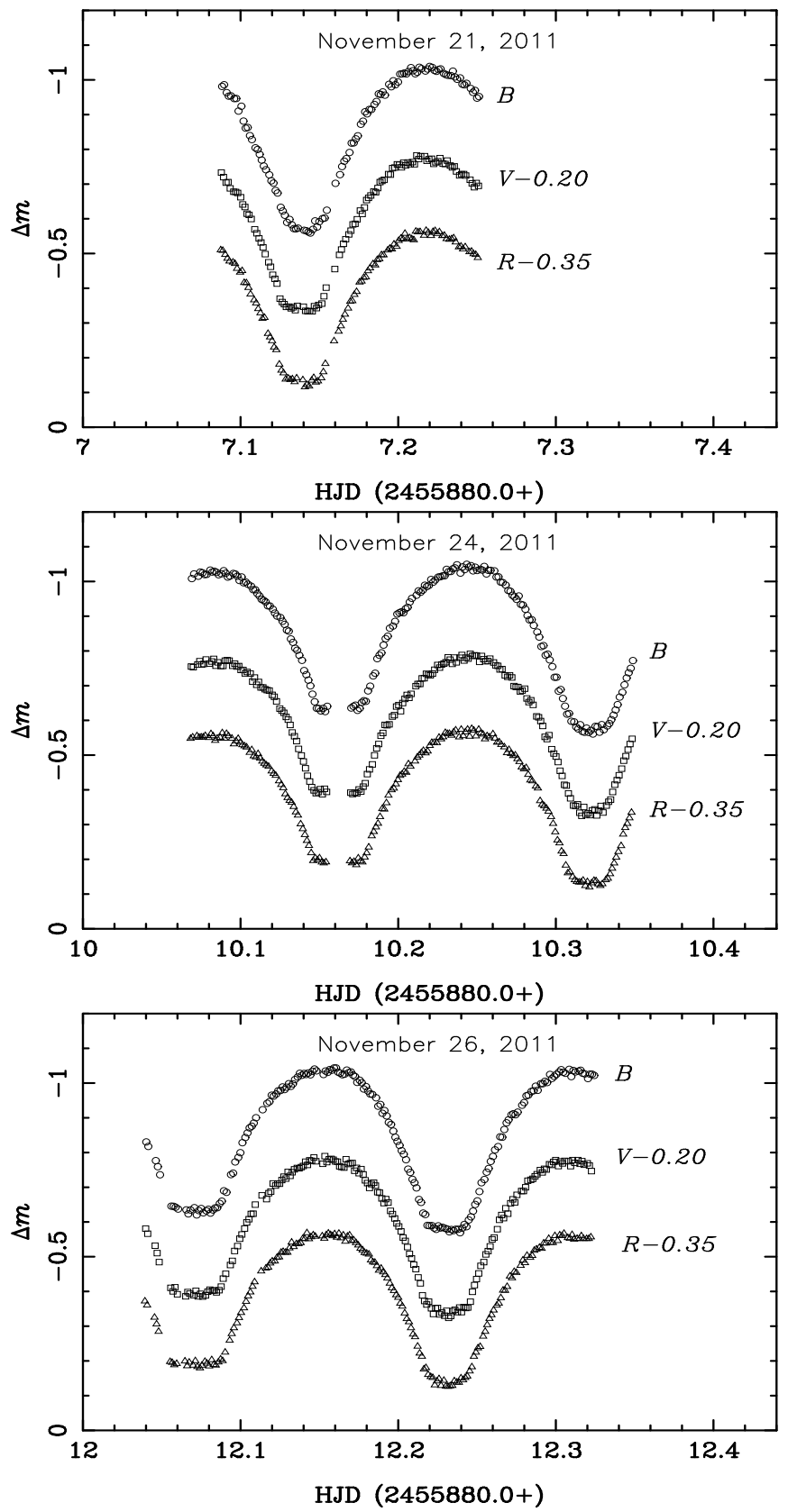

Figure 1: The observed light curves in $B$ (open dots), $V$ (squares) and $R$ (triangles) filter bands for BO Ari on November 21 (top), 24 (middle) and 26 (bottom), 2011. 
time of 30 seconds. A total of 1446 individual observations were obtained for all three filter bands (482 observations per filter band). Data reduction and $B V R$ differential magnitude measurements were made with the IRAF package.

GSC $1761-1578\left(\operatorname{RA}(2000)=02^{\mathrm{h}} 12^{\mathrm{m}} 11^{\mathrm{s}} .18\right.$, Dec. $\left.(2000)=27^{\circ} 10^{\prime} 19^{\prime \prime} .8\right)$ and TYC 1761-2130-1 $\left(\mathrm{RA}(2000)=02^{\mathrm{h}} 11^{\mathrm{m}} 53^{\mathrm{s}} .48\right.$, Dec. $\left.(2000)=27^{\circ} 15^{\prime} 34^{\prime \prime} .5\right)$ were used as comparison and check stars, respectively. The observed $B V R$ light curves for the three nights covered five times of minimum light, as shown in Fig. 1. All minimum light times were found by phase shifting by about half an orbital period with respect to previous minimum light times. The amplitudes were about $0.47,0.45$ and $0.44 \mathrm{mag}$, for the $B$-, $V$ - and $R$-bands, respectively.

\section{Orbital period analysis}

This study obtained three primary and two secondary minima. In order to analyze the orbital period change of BO Ari, orbital phases of all available minimum light times were shifted with 0.5 periods from the original sources to correspond with the data in this study as listed in column 4 of Table 1. Early minimum light times were found with nearly equal depths of primary and secondary eclipses such the primary minima could not always be distinguished from secondary minima. This is why we applied a 0.5 phase shift on previous data. This study found that the primary minima had become clearly deeper than the secondary ones but this did not always agree with the previous series of minimum light. After phase shifting, all minimum light times at present were fitted with a linear least-squares as shown in Eq. (1). The result gave a revised orbital period of $0.3181945( \pm 0.0000002) \mathrm{d}$.

$$
\text { Min.I }=\text { HJD2455887.1405 }( \pm 0.0006)+0.3181945( \pm 0.0000002) \times E
$$

The orbital period of $\mathrm{BO}$ Ari in this study was shorter than the value obtained by Acerbi, Barani and Martignoni (2011). The $(O-C)$ residuals were calculated by using the Eq. (1), and listed in the Table 1. Quadratic least-squares fitting was applied to the $(O-C)$ as shown in Eq. (2) with the rms of the $(O-C)$ curve residuals of about $0.00134 \mathrm{~d}$. The $(O-C)$ curve and corresponding residuals are plotted in Fig. 2. The fitting solution indicated that a long-term orbital period decrease of $\mathrm{BO}$ Ari at a rate of

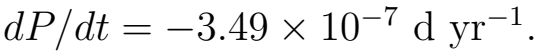




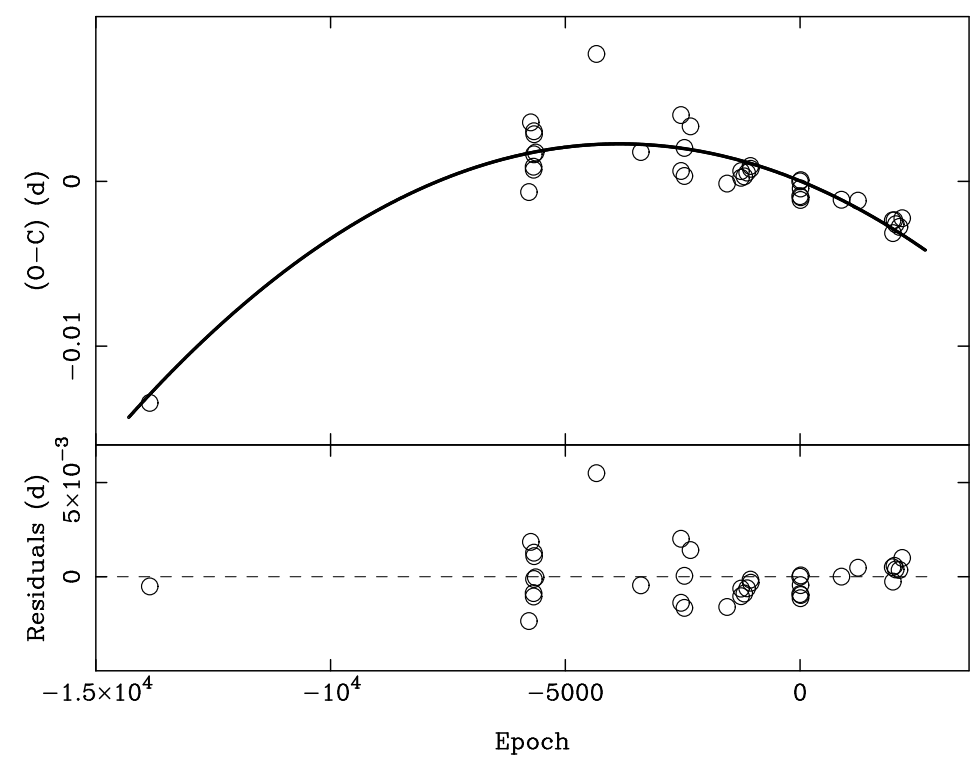

Figure 2: The $(O-C)$ curve (upper panel) and the residuals (lower panel) for BO Ari, corresponding to the Eq. (2).

$(O-C)=0.0000( \pm 0.0003)-1.17( \pm 0.12) \times 10^{-6} E-1.52( \pm 0.12) \times 10^{-10} E^{2}$

As shown by the residuals in Fig. 2, it seems that there may be a cyclic variation, which can be produced by a LTE due to the presence of a third body in the system (Irwin, 1952; Pribulla and Rucinski, 2006). This cyclic period change can be analyzed with the following formula:

$$
\text { Min.I }=T_{0}+P E+A E^{2}+\tau_{3}
$$

where $T_{0}+P E+A E^{2}$ includes the quadratic long-term period change. $T_{0}$ and $P$ are revised initial epoch and period, and $A$ is a half of the period change per cycle. $\tau_{3}$ is added for the LTE due to the third companion (e.g. Lee et al., 2011; Zhu, Qian and Li, 2011), as follows:

$$
\begin{aligned}
\tau_{3} & =K\left[\frac{1-e^{2}}{1+e \cos \nu} \sin (\nu+\omega)+e \sin \omega\right] \\
& =K\left[\sqrt{1-e^{2}} \sin E^{*} \cos \omega+\cos E^{*} \sin \omega\right]
\end{aligned}
$$


Table 1: All available times of light minimum for BO Ari.

\begin{tabular}{lrrrrr}
\hline \hline HJD & Type 1 & Ref. & Type 2 & Epoch & $(O-C)$ \\
1 & 2 & 3 & 4 & 5 & 6 \\
\hline 2451479.6566 & II & {$[1]$} & I & -13851.5 & -0.0134 \\
2454049.4078 & II & {$[2]$} & I & -5775.5 & -0.0006 \\
2454062.4580 & II & {$[3]$} & I & -5734.5 & 0.0036 \\
2454080.4333 & I & {$[3]$} & II & -5678.0 & 0.0009 \\
2454081.3877 & I & {$[3]$} & II & -5675.0 & 0.0007 \\
2454083.2978 & I & {$[3]$} & II & -5669.0 & 0.0016 \\
2454083.4583 & II & {$[3]$} & I & -5668.5 & 0.0030 \\
2454084.4127 & II & {$[2]$} & I & -5665.5 & 0.0029 \\
2454095.3893 & I & {$[3]$} & II & -5631.0 & 0.0018 \\
2454507.2980 & II & {$[4]$} & I & -4336.5 & 0.0077 \\
2454808.3040 & II & {$[4]$} & I & -3390.5 & 0.0018 \\
2455080.3625 & II & {$[5]$} & I & -2535.5 & 0.0040 \\
2455080.5182 & I & {$[6]$} & II & -2535.0 & 0.0006 \\
2455103.4279 & I & {$[6]$} & II & -2463.0 & 0.0003 \\
2455103.5887 & II & {$[6]$} & I & -2462.5 & 0.0020 \\
2455144.3189 & II & {$[6]$} & I & -2334.5 & 0.0033 \\
2455392.5071 & II & {$[5]$} & I & -1554.5 & -0.0001 \\
2455487.3294 & II & {$[7]$} & I & -1256.5 & 0.0002 \\
2455487.4889 & I & {$[7]$} & II & -1256.0 & 0.0006 \\
2455509.4440 & I & {$[7]$} & II & -1187.0 & 0.0003 \\
2455528.3768 & II & {$[7]$} & I & -1127.5 & 0.0005 \\
2455551.2872 & II & {$[7]$} & I & -1055.5 & 0.0009 \\
2455553.1962 & II & {$[7]$} & I & -1049.5 & 0.0008 \\
2455887.1396 & I & {$[8]$} & I & 0.0 & -0.0009 \\
2455890.1622 & II & {$[8]$} & II & 9.5 & -0.0011 \\
2455890.3220 & I & {$[8]$} & I & 10.0 & -0.0004 \\
2455892.0716 & II & {$[8]$} & II & 15.5 & -0.0010 \\
2455892.2317 & I & {$[8]$} & I & 16.0 & 0.0001 \\
2456167.9460 & II & {$[9]$} & I & 882.5 & -0.0011 \\
2456279.6322 & II & {$[10]$} & I & 1233.5 & -0.0012 \\
2456514.9358 & I & {$[11]$} & II & 1973.0 & -0.0024 \\
2456516.5260 & I & {$[12]$} & II & 1978.0 & -0.0031 \\
2456526.8681 & II & {$[11]$} & I & 2010.5 & -0.0024 \\
2456535.9364 & I & {$[11]$} & II & 2039.0 & -0.0026 \\
2456560.9145 & II & {$[11]$} & I & 2117.5 & -0.0028 \\
2456579.6885 & II & {$[11]$} & I & 2176.5 & -0.0022 \\
2456579.8475 & I & {$[11]$} & II & 2177.0 & -0.0023 \\
\hline & & & & &
\end{tabular}

Notes: Column 1: HJD at light minimum. Column 2: types of minimum from original sources. Column 3: references for the sources are as follow: [1] Nicholson and Varley (2006); [2] Martignoni (2011); [3] Acerbi, Barani and Martignoni (2011); [4] Paschke (2009); [5] Demircan et al. (2011); [6] Gökay et al. (2010); [7] Gökay et al. (2012); [8] This study; [9] Nelson (2013); [10] Diethelm (2013); [11] Nelson (2014); [12] Paschke (2014). Column 4: types of minimum with phase shifted of 0.5 periods to correspond with the data in this study. Column 5: epoch. Column 6: $(O-C)$, corresponding to the Eq. (2). 
where $K=a_{12} \sin i_{3} / c$ is the amplitude of the cyclic variation. The parameters $a_{12}, i_{3}, e$ and $\omega$ are the semi-major axis, inclination, eccentricity and longitude of the periastron of the binary's orbit around the mass center of the triple system, respectively, and $\nu$ is the true anomaly of the position of the binary's mass center in the orbit. $E^{*}$ is the eccentric anomaly, computed together with Eq. (5) (e.g. Irwin, 1952; Zhu, Qian and Li, 2011; Kriwattanawong and Poojon, 2015):

$$
2 \pi \frac{t-T}{P_{3}}=M=E^{*}-e \sin E^{*}
$$

where $M$ is the mean anomaly, $t$ is the time of minimum light, $T$ is the time of periastron passage for the third body and $P_{3}$ is the orbital period of the third companion in the system. If a cyclic period change is included in the model due to the LTE with an orbital period of $5.54 \mathrm{yrs}$, the $\mathrm{rms}$ of the residuals is $0.00116 \mathrm{~d}$, which is not much less than for the solution without LTE in Eq. (2) which is $0.00134 \mathrm{~d}$. These $\mathrm{rms}$ values of the residuals are of the same order as the amplitude of $0.0011 \mathrm{~d}$ of this cyclic variability. Therefore this cyclic variability is not detected at a significant level (in that case the amplitude should have been $>3$ times the $r m s$ values). We therefore conclude that there is no evidence for the presence of a third component.

\section{Photometric Solutions}

In this study, the secondary minima were slightly higher than the primary ones about $0.06 \mathrm{mag}$. for all $B V R$ bands as shown in Fig. 1. The primary massive star was eclipsed by the less massive star at the primary minimum, indicating that this contact binary is an A-type system, same as identified by Acerbi, Barani and Martignoni (2011). For the A-type contact binary, the more massive component is assigned to be star 1 and the other component is assigned as star 2. The color $(B-V)_{\text {Tycho }}=0.581$ for BO Ari was collected from the Tycho 2 catalogue (Høg et al., 2000). Since, the relation $(B-V)_{\text {Johnson }}=0.85(B-V)_{\text {Tycho }}$ (e.g. Gürol et al., 2011; Kriwattanawong and Poojon, 2014), the $(B-V)$ color of BO Ari was obtained to be 0.494. This indicates that the primary should be a late F-type main sequence star, with an effective temperature of about $6250 \mathrm{~K}$ (Cox, 2000). Coincidently, the effective temperature of BO Ari was calibrated by using spline method, and reported to be $6252 \mathrm{~K}$ (Ammons et. al., 2006). Thus, the effective temperature of the star $1, T_{1}$, was assumed as $6250 \mathrm{~K}$. This is about $1000 \mathrm{~K}$ hotter 
Table 2: Parameters for the period change with and without the LTE.

\begin{tabular}{|c|c|c|}
\hline \multirow[t]{2}{*}{$\overline{\text { Parameters }}$} & \multicolumn{2}{|c|}{ Values } \\
\hline & Quadratic & Quadratic plus LTE \\
\hline$\overline{T_{0}(\mathrm{~d})}$ & $2455877.1405( \pm 0.0006)$ & $2455877.1405( \pm 0.0006)$ \\
\hline$P(\mathrm{~d})$ & $0.3181933( \pm 0.0000002)$ & $0.3181932( \pm 0.0000002)$ \\
\hline$A(\mathrm{~d})$ & $-1.52( \pm 0.12) \times 10^{-10}$ & $-1.59( \pm 0.12) \times 10^{-10}$ \\
\hline$d P / d t\left(\mathrm{~d} \mathrm{yr}^{-1}\right)$ & $3.49( \pm 0.28) \times 10^{-7}$ & $3.65( \pm 0.28) \times 10^{-7}$ \\
\hline$\overline{K(\mathrm{~d})}$ & - & $0.0011( \pm 0.0002)$ \\
\hline$a_{12} \sin i_{3}(\mathrm{AU})$ & - & $0.19( \pm 0.03)$ \\
\hline$e$ & - & $0.24( \pm 0.06)$ \\
\hline$\omega\left({ }^{\circ}\right)$ & - & $43.0( \pm 3.7)$ \\
\hline$P_{3}(\mathrm{yr})$ & - & $5.54( \pm 0.27)$ \\
\hline$T(\mathrm{~d})$ & - & $2456373.2( \pm 78.6)$ \\
\hline rms of residuals $(\mathrm{d})$ & 0.00134 & 0.00116 \\
\hline
\end{tabular}

Notes: Column 1: fitted parameters. Column 2: values of the parameters, corresponding to the quadratic least-squares without the LTE, Eq. (2). Column 3: values of the parameters, corresponding to the quadratic least-squares with the LTE, Eqs. (3) - (5).

that the value assumed in previous study by Acerbi, Barani and Martignoni (2011). The Wilson-Devinney (W-D) method (Wilson and Devinney, 1971; Wilson, 1979, 1990; Wilson and van Hamme, 2003) was used to derive the photometric solution for BO Ari. The W-D code was used in mode 3, with more fixed parameters as follow: the gravity darkening exponents of $g_{1}=g_{2}$ $=0.32$ (Lucy, 1967), the bolometric albedo coefficients of $A_{1}=A_{2}=0.50$ (Rucinski, 1973), and the limb darkening coefficients of $x_{1 B}=x_{2 B}=0.877$, $x_{1 V}=x_{2 V}=0.671$ and $x_{1 R}=x_{2 R}=0.514$ for the $B V R$ filter bands, corresponding to the temperature $T_{1}$ (van Hamme, 1993).

In order to complete the W-D fitting, we adjusted the following parameters: the orbital inclination, $i$, the mass ratio, $q$, the surface potential of the components, $\Omega_{1}=\Omega_{2}$, the temperature of the star $2, T_{2}$, and the monochromatic luminosities for the $B V R$ filter bands of the star $1, L_{1}$. The relative luminosities of the star $2, L_{2}$, for all bands were automatically estimated by the W-D code, following the stellar atmosphere model of Kurucz (1993).

The photometric parameters were obtained for the minimum sum of the squared residuals, $\Sigma(O-C)^{2}=0.0124$, resulting in a mass ratio of $q=0.1754$ 
Table 3: Photometric parameters for BO Ari.

\begin{tabular}{lrr}
\hline \hline Parameters & \multicolumn{2}{c}{ Values } \\
\cline { 2 - 3 } & Previous study ${ }^{\dagger}$ & This study \\
\hline$q$ & $0.1889( \pm 0.0022)$ & $0.1754( \pm 0.0016)$ \\
$T_{1}(K)$ & 5307 & 6250 \\
$T_{2}(K)$ & $5254( \pm 12)$ & $6409( \pm 17)$ \\
$i\left(^{\circ}\right)$ & $80.96( \pm 0.03)$ & $81.12( \pm 0.24)$ \\
$\Omega_{1}=\Omega_{2}$ & $2.1340( \pm 0.0033)$ & $2.1391( \pm 0.0020)$ \\
$g_{1}=g_{2}$ & 0.30 & 0.32 \\
$A_{1}=A_{2}$ & 0.50 & 0.50 \\
$x_{1 B}=x_{2 B}$ & 0.411 & 0.877 \\
$x_{1 V}=x_{2 V}$ & 0.736 & 0.671 \\
$x_{1 R}=x_{2 R}$ & - & 0.514 \\
$L_{1 B} /\left(L_{1 B}+L_{2 B}\right)$ & $0.785( \pm 0.001)$ & $0.8017( \pm 0.0172)$ \\
$L_{1 V} /\left(L_{1 V}+L_{2 V}\right)$ & $0.787( \pm 0.001)$ & $0.8075( \pm 0.0124)$ \\
$L_{1 R} /\left(L_{1 R}+L_{2 R}\right)$ & & $0.8109( \pm 0.0087)$ \\
$r_{1}$ (pole) & $0.5084( \pm 0.0008)$ & $0.5044( \pm 0.0007)$ \\
$r_{1}$ (side) & $0.5606( \pm 0.0012)$ & $0.5539( \pm 0.0013)$ \\
$r_{1}$ (back) & $0.5884( \pm 0.0016)$ & $0.5780( \pm 0.0017)$ \\
$r_{2}$ (pole) & $0.2488( \pm 0.0010)$ & $0.2330( \pm 0.0017)$ \\
$r_{2}$ (side) & $0.2618( \pm 0.0012)$ & $0.2435( \pm 0.0022)$ \\
$r_{2}$ (back) & $0.3190( \pm 0.0032)$ & $0.2848( \pm 0.0038)$ \\
Spot Colatitude $\left(^{\circ}\right)$ & $* * 90$ & $* 74.48( \pm 3.04)$ \\
Spot Longitude $\left(^{\circ}\right)$ & $* * 260.77( \pm 5.2)$ & $* 315.13( \pm 3.24)$ \\
Spot Radius $\left(^{\circ}\right)$ & $* * 29.96( \pm 1.6)$ & $* 10.31( \pm 1.69)$ \\
Temperature Factor & $* * 0.9021( \pm 0.008)$ & $* 0.95( \pm 0.02)$ \\
$\Sigma(O-C)^{2}$ & 0.0132 & 0.0124 \\
$f$ (\%) & $58.7( \pm 0.2)$ & $27.72( \pm 2.37)$ \\
\hline
\end{tabular}

Notes: $\dagger^{\dagger}$ Acerbi, Barani and Martignoni (2011).

* Parameters of a starspot on the primary star.

** Parameters of a starspot on the secondary star.

$( \pm 0.0018)$. This mass ratio was slightly less than the value obtained by Acerbi, Barani and Martignoni (2011). The inclination of the orbit was 


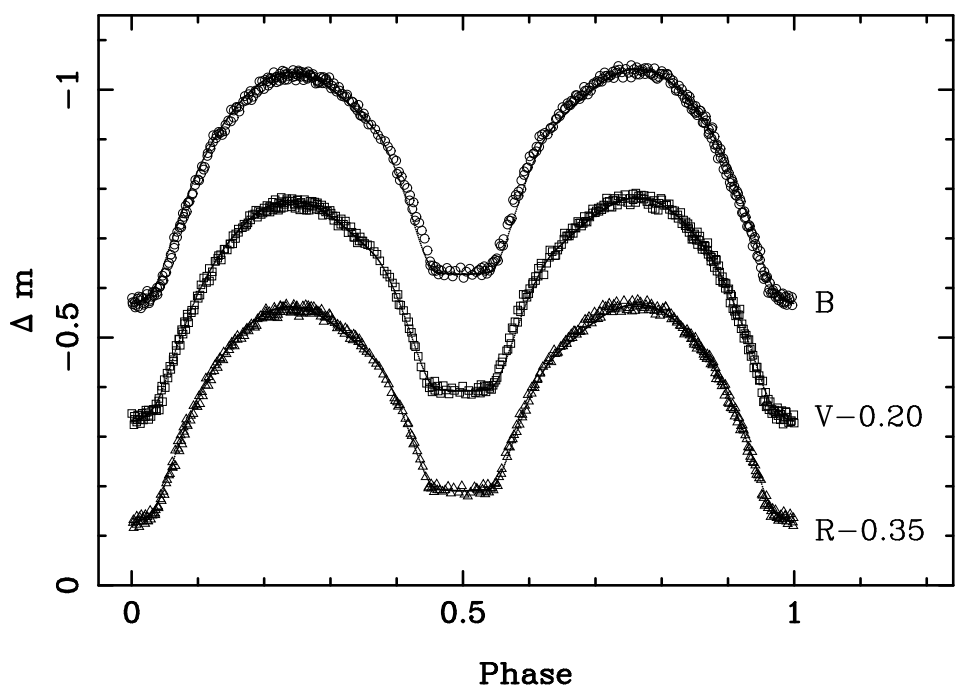

Figure 3: The observed light curves in $B$ (open dots), $V$ (squares) and $R$ (triangles) filter bands and the theoretical light curves (solid lines) versus orbital phase for BO Ari.

$i=81.21^{\circ}$, confirming total eclipse on the eclipsing pair, strengthening the reliability of the mass ratio. A starspot was added on the primary component, with temperature factor of 0.95 , which can explain a small asymmetry of the light curves due to the O'Connell effect. The overcontact degree was found to be $27.72 \%( \pm 2.37 \%)$, which is about half the degree derived by Acerbi, Barani and Martignoni (2011). This difference could be due to the fixed effective temperature, $T_{1}$, and the corresponding limb darkening coefficients $x_{1}$ and $x_{2}$ which were assumed to have different values. The theoretical light curves are plotted overlying on the observed ones in Fig. 3, and all photometric elements are listed in Table 3.

\section{Discussion and Conclusions}

The $B V R$ observations in this study were obtained during three nights in 2011, covering five minimum light times. The light curves confirmed that BO Ari is an A-type, same as firstly reported by Acerbi, Barani and Martignoni (2011). The revised orbital period is obtained to be 0.3181933 $( \pm 0.0000002)$. The $(O-C)$ residuals were first examined by using the quadratic least-squares fit, showing a downward parabolic trend line, that 
indicates the long-term orbital period decrease. The orbital period of BO Ari decreases at a rate of $d P / d t=-3.49 \times 10^{-7} \mathrm{~d} \mathrm{yr}^{-1}$.

The $(B-V)$ color of $\mathrm{BO}$ Ari was estimated to be 0.494 , corresponding to an effective temperature of $T_{1}=6250 \mathrm{~K}$ for the primary star (Cox, 2000). With this result, the effective temperature $T_{1}$ and the corresponding limb darkening coefficients of $x_{1}$ and $x_{2}$ for $B V R$ filter bands (van Hamme, 1993) are quite far from the values in the previous study (Acerbi, Barani and Martignoni, 2011). The photometric elements were derived for mass ratio $q=0.1754$ with the rms of the residuals, $\Sigma(O-C)^{2}=0.0124$, while the contact degree was found to be $f=27.72 \%$. The mass ratio confirms that $\mathrm{BO}$ Ari is a low mass ratio contact system. Our results indicate that contact configuration of BO Ari is not very deep. More observations are required to confirm these parameters.

The system shows a long-term period decrease, implying mass transfer from the more massive primary component to the less massive one. The rate of mass transfer $\dot{m}_{1} / m_{1}$ can be estimated, under the conservative assumption (Singh and Chaubey, 1986; Pribulla, 1998) by:

$$
\frac{\dot{P}}{P}=3\left(\frac{m_{1}}{m_{2}}-1\right) \frac{\dot{m}_{1}}{m_{1}}
$$

The resulting rate of mass transfer is $\dot{m}_{1} / m_{1}=-7.77 \times 10^{-8} \mathrm{yr}^{-1}$. With the mass transfer, the inner and outer critical Roche lobes will shrink and cause the contact degree to increase, similar to the case of WZ Cep (Zhu and Qian, 2009), AH Tau (Yang et al., 2010), and GV Leo (Kriwattanawong and Poojon, 2013). Therefore, the low mass ratio contact binary, BO Ari could evolve into a system with deeper contact.

\section{Acknowledgments}

We acknowledge support by Chiang Mai University. Observations were obtained, using the 0.5-m telescope at Sirindhorn Observatory, Chiang Mai University. This research has made use of the SIMBAD online database, operated at CDS, Strasbourg, France and the NASA's Astrophysics Data System (ADS), operated by the Smithsonian Astrophysical Observatory (SAO) under a NASA grant.

\section{References}

Acerbi, F., Barani, C., Martignoni, M., 2011. RAA 11, 843. 
Ammons, S. M., Robinson, S. E., Strader, J., Laughlin, G., Fischer, D., Wolf, A., 2006. ApJ 638, 1004A.

Binnendijk, L., 1970. Vistas in Astronomy 12, 217.

Cox, A. N., 2000. Allen's Astrophysical Quantities, fourth ed. Springer, New York, pp. 388.

Christopoulou, P.-E., Parageorgiou, A., Chrysopoulos, I., 2011. AJ 142, 99.

Diethelm, R., 2013. IBVS 6042, 1.

Demircan, Y., Gürol, B., Gökay, G., Terzioğlu, Z., Saral, G., Gürsoytrak, H., Okan, A., Demirhan, U., Coker, D., Derman, E., 2011. IBVS 5965, 1.

Gazeas, K. D., Niarchos, P. G., 2006. MNRAS 370, L29.

Gökay, G., Demircan, Y., Terzioğlu, Z., Gürsoytrak, H., Okan, A., Çoker, D., Saral, G., Gürol, B., Derman, E., 2010. IBVS 5922, 1.

Gökay, G., Demircan, Y., Gürsoytrak, H., Terzioğlu, Z., Okan, A., Doğruel, M. B., Saral, G., Cerit, S., Şemuni, M., Kiliç, Y., Çoker, D., Derman, E., Gürol, B., 2012. IBVS 6039, 1.

Gürol, B., Derman, E., Saguner, T., Gürsoytrak, S. H., Terzioğlu, Z., Gökay, G., Demircan, Y., Okan, A., Saral, G., 2011. NewA 16, 242.

Høg, E., Fabricius, C., Makarov, V. V., Urban, S., Corbin, T., Wycoff, G., Bastian, U., Schwekendiek, P., Wicenec, A., 2000. A\&A 355, L27.

Irwin, J. B., 1952. ApJ 116, 211.

Kriwattanawong, W., Poojon, P., 2013. RAA 13, 1330.

Kriwattanawong, W., Poojon, P., 2014. NewA 28, 23.

Kriwattanawong, W., Poojon, P., 2015. NewA 36, 50.

Kurucz, R. L., 1993. A New Opacity-Sampling Model Atmosphere Program for Arbitrary Abundances, in: Dworetsky, M. M., Castelli, F., Faraggiana, R. (Eds.), Peculiar versus Normal Phenomena in A-type and Related Stars. ASP Conf. Ser. 44, 87. 
Lee J. W., Kim, S.-L., Lee, C.-U., Kim, H.-I., Park, J.-H., Hinse, T. C., 2011. AJ 142, 12.

Li, K., Qian, S.-B., 2013. NewA 25, 12.

Liu, N.-P., Qian, S.-B., Liao, W.-P., He, J.-J., Zhao, E.-G., Liu, L., 2014. RAA 14, 1157.

Lucy, L. B., 1967. Z. Astrophys. 65, 89.

Lucy, L. B., 1976. ApJ 205, 208.

Martignoni, M., 2011. IBVS 6006, 1.

Nicholson, M, Varley, H., 2006. IBVS 5700, 1.

Nelson, R. H., 2013. IBVS 6050, 1.

Nelson, R. H., 2014. IBVS 6092, 1.

Paschke A., 2009. QEJV 116, 1.

Paschke A., 2014. QEJV 162, 1.

Pribulla, T., 1998. Contrib. Astron. Obs. Skalnaté Pleso 28, 101.

Pribulla, T., Rucinski, S. M., 2006. ApJ 131, 2986.

Robertson, J. A., Eggleton, P. P., 1977. MNRAS 179, 359.

Rucinski, S. M., 1973. Acta Astron. 23, 79.

Rucinski, S. M., 1982. A\&A 112, 273.

Rucinski, S. M., Duerbeck, H. W., 1997. PASP 109, 1340.

Singh, M., Chaubey, U., 1986. Ap\&SS 124, 389.

Stępień, K., 2006. Acta Astron. 56, 199.

Terrell, D., Gross, J., Cooney, W. R., 2012. AJ 143, 99.

van Hamme, W., 1993. AJ 106, 2096.

Vilhu, O., 1981. Ap\&SS 78, 401. 
Wilson, R. E., 1979. ApJ 234, 1054.

Wilson, R. E., 1990. ApJ 356, 613.

Wilson, R. E., Devinney, E. J., 1971. ApJ 166, 605.

Wilson, R. E., van Hamme, W., 2003. Computing Binary Stars Observables, the 4th edition of the W-D program.

Yakut, K., Eggleton, P. P., 2005. ApJ 629, 1055.

Yang, Y.-G., 2011. RAA 11, 181.

Yang, Y.-G., Wei, J.-Y., Kreiner, J. M., Li, H.-L., 2010. AJ 139, 195.

Zhu, L. Y., Qian, S. B., 2009. AJ, 138, 2002.

Zhu, L., Qian, S.-B., Li, L., 2011. PASJ, 64, 94. 